\title{
Duplex DNA is Weakened in Nanoconfinement
}

Sagun Jonchhe, Shankar Pandey, Deepak Karna, Pravin Pokhrel, Yunxi Cui, Shubham Mishra, Hiroshi Sugiyama*, Masayuki Endo*, Hanbin Ma**

\section{Supporting Information}

\section{Table of Contents}

1. Synthesis of the DNA origami nanocages that contain hairpin hosting DNA fragments.

2. Synthesis strategy for the single-stranded DNA containing the bcl-2 hairpin forming sequence.

3. Synthesis strategy for the single-stranded DNA that contains a telomeric G-quadruplex sequence and a telomeric i-motif sequence.

4. Characterization of the single molecular DNA nanocage construct by AFM.

5. Unfolding/refolding force and change-in-contour-length measurements.

6. Percentage populations of the hairpin and tetraplex structures..

7. Unfolding force versus extension plots.

8. Expected change-in-contour-length $(\Delta L)$.

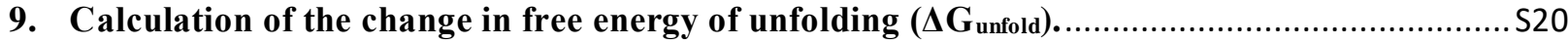

10. Estimation of the change in free energy of hairpin unfolding using mfold ${ }^{\circledR}$.......................... 222

11. Energy stored in the unfolding pathway of the bcl-2 hairpin...........................................S23

12. Point spread function used to convert species populations to relative free energies............... $S 23$

13. Coarse-grained molecular dynamics (MD) simulation of bcl-2 hairpin inside the nanocages. S24

14. Quantitation of factors in the reduced stability of the duplex DNA.....................................S25

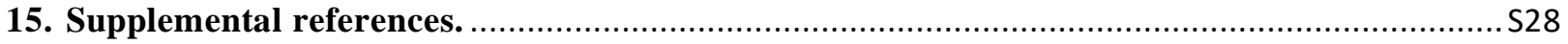


1. Synthesis of the DNA origami nanocages that contain hairpin hosting DNA fragments.

Table S1. Plasmid, restriction enzyme, and primer strand for preparation of nanocages

\begin{tabular}{|c|c|c|}
\hline Nanocages & $\begin{array}{c}\text { Plasmid, Restriction } \\
\text { enzyme, Length }\end{array}$ & \multicolumn{1}{c|}{ Complementary primer strands } \\
\hline $15 \times 15 \mathrm{~nm}$ & p8064, PacI/ Bg1II, & 5'-TAAGGGAAAATTAATTAATAGCGACGAT \\
$(7 \times 7 \mathrm{HB})$ & $3624 \mathrm{nts}$ & 5'- TAGCCTTTGTAGATCTCTCAAAAATA \\
\hline
\end{tabular}




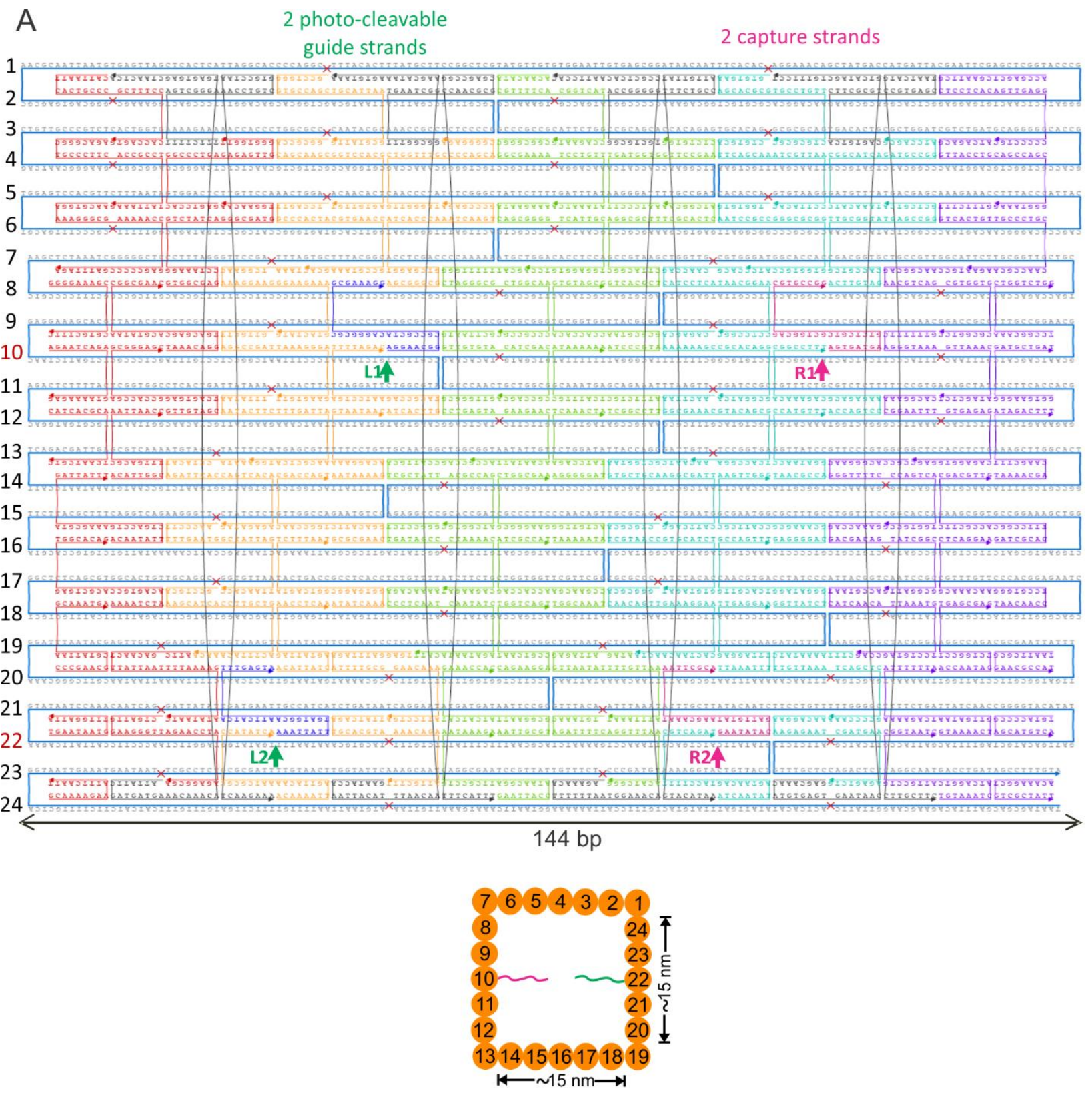

B

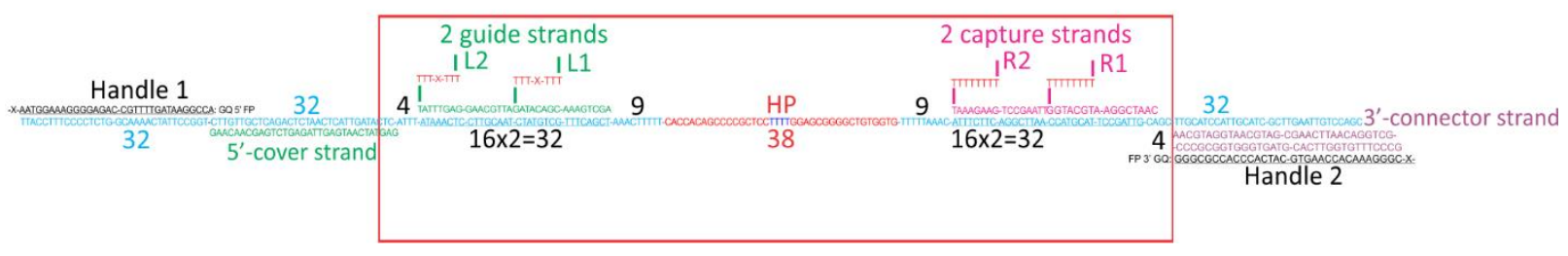

Figure S1. Design of the $15 \times 15 \mathrm{~nm}(7 \times 7$ Helical Bundles, HB) nanocage. (A) caDNAno design of the 15×15 nm DNA origami nanocage that contains bcl-2 hairpin forming strand. L1 and L2 
represent the connection sites of the photo-cleavable guide strands while $\boldsymbol{R} \mathbf{1}$ and $\boldsymbol{R} 2$ represent the connection sites of the capture strands to link the hairpin hosting strand to the wall of the nanocage. A fragment of p8064 (3624 nts) was used as a template strand. Diagram shown at the bottom is the side view of the DNA nanocage. Each red cross mark is deletion of a base compared to previous design ${ }^{S 1}$ to adjust the pitch close to $10.5 \mathrm{bp} / \mathrm{turn}$. (B) Detailed sequence of the 224-nt bcl-2 hairpin (HP) hosting strand. The tethering sites to the DNA nanocage (L1, L2, R1, R2) and the attachment sites to the dsDNA handles (Handle 1 and Handle 2) are depicted. Each number in $(B)$ represents the length of a specific DNA fragment. The structure of photo-cleavable $(X)$ is shown in Table $S 2$. 


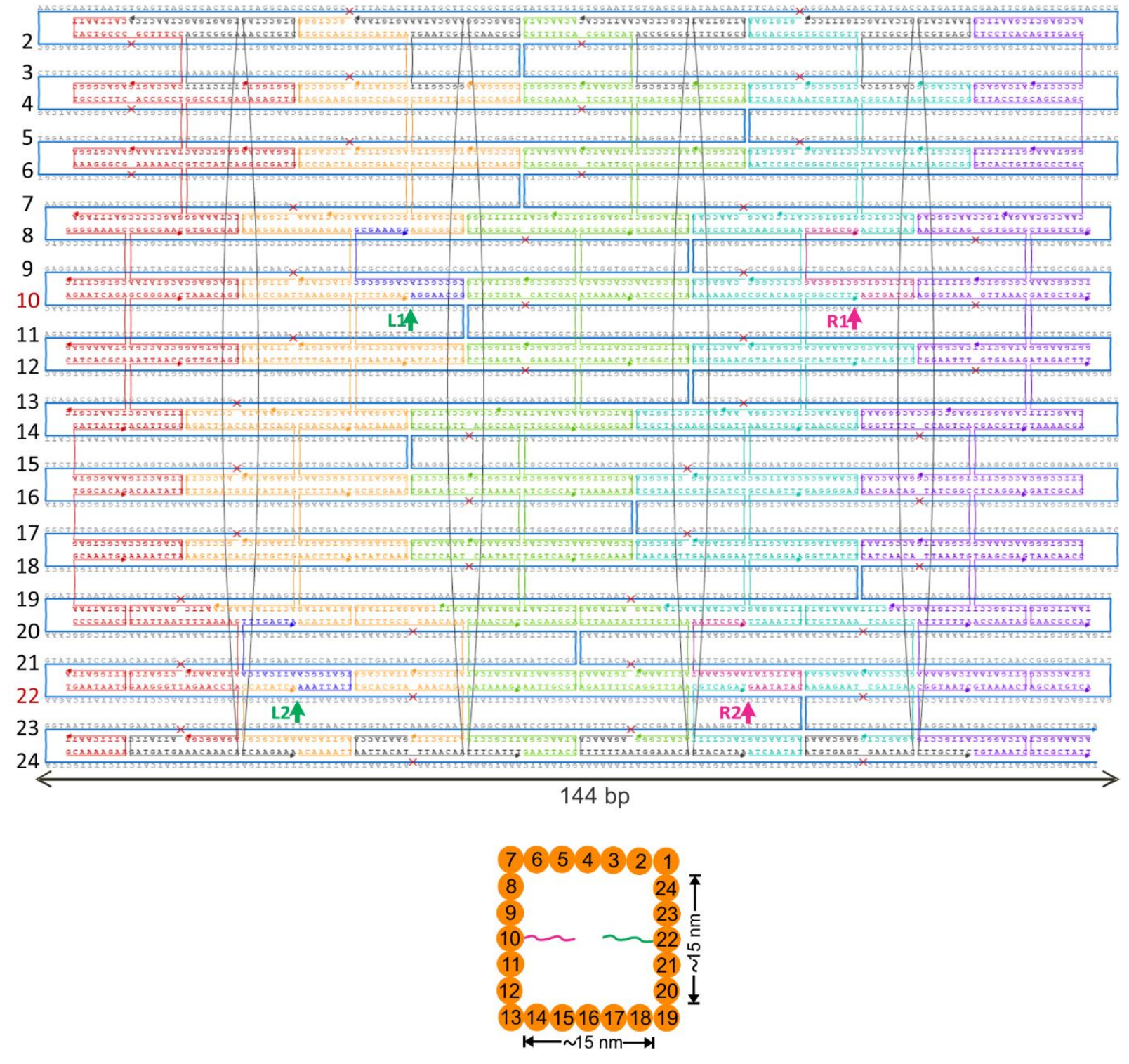

B

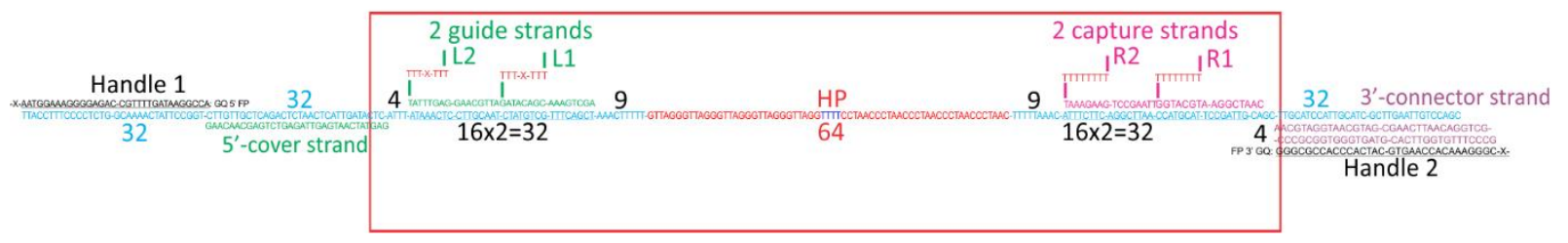

Figure S2. Design of the $15 \times 15 \mathrm{~nm}(7 \times 7$ Helical Bundles, HB) nanocage. (A) caDNAno design of the $15 \times 15 \mathrm{~nm}$ DNA origami nanocage that contains hairpin forming strand, with i-motif (iM) and G-quadruplex $(G Q)$ forming sequences in complementary strands of the hairpin stem. L1 and $\boldsymbol{L} 2$ represent the connection sites of the photo-cleavable guide strands while $\boldsymbol{R} 1$ and $\boldsymbol{R} 2$ represent the connection sites of the capture strands to link the hairpin hosting strand to the wall of the 
nanocage. A fragment of p8064 (3624 nts) was used as a template strand. Diagram shown at the bottom is the side view of the DNA nanocage. Each red cross mark is deletion of a base compared to previous design ${ }^{S 1}$ to adjust the pitch close to $10.5 \mathrm{bp} / \mathrm{turn}$. (B) Detailed sequence of the 250-nt hairpin (HP) hosting strand. The tethering sites to the DNA nanocage (L1, L2, R1, R2) and the attachment sites to the dsDNA handles (Handle 1 and Handle 2) are depicted. Each number in (B) represents the length of a specific DNA fragment. The structure of photo-cleavable $(X)$ is shown in Table S2.

Table S2. DNA staple sequences used to synthesize $15 \mathrm{~nm} \times 15 \mathrm{~nm}$ DNA origami nanocage. Green and pink colored staple sequences containing 16-nt overhangs are used to hybridize with guide and capture strands, respectively. Underlined sequences in the guide and capture strands represent the respective staple sequences that hybridize with the overhangs mentioned above. The red "TTTTTTTT" and "TTTXTTT" sequences in the respective capture and guide strands represent the linkers between the nanocage and the HP strand, here ' $X$ ' in the guide strands depicts a photo-cleavable group,

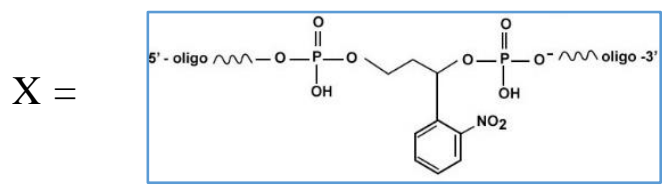

\begin{tabular}{|c|c|c|}
\hline Staple Name & Sequence & Length \\
\hline $7 \mathrm{X} 7 \mathrm{NC} 01$ & CATTAATTCACTGCCCGCTTTCCCACCAGTG & 31 \\
\hline $7 \mathrm{X} 7 \mathrm{NC} 02$ & GCCTGGGGTGCCAGCTGCATTAAGCGTATTG & 31 \\
\hline $7 \mathrm{X} 7 \mathrm{NC} 03$ & ACAACATACGTTTTCACGGTCATCCAGAATG & 31 \\
\hline $7 \mathrm{X} 7 \mathrm{NC} 04$ & GTGTGAAAGCACGCGTGCCTGTTCTGCGCGC & 31 \\
\hline $7 \mathrm{X} 7 \mathrm{NC} 05$ & ACCGAGCTCGAATTCGCTCCTCACAGTTGAGGCCCCCTGC & 40 \\
\hline $7 \mathrm{X} 7 \mathrm{NC} 06$ & AGACGGGCTGCCCTTCACCGCCTTATTAAAG & 31 \\
\hline $7 \mathrm{X} 7 \mathrm{NC} 07 \mathrm{C}$ & TTTCTTTTAGTCGGGAAACCTGTCGTGCCTAATCAAGAAA & 40 \\
\hline $7 \mathrm{X} 7 \mathrm{NC} 08$ & GGCGCCACAGCAAGCGGTCCACGAGTGTTGT & 31 \\
\hline $7 \mathrm{X} 7 \mathrm{NC} 09 \mathrm{C}$ & GGCGGTTTTGAATCGGCCAACGCGCGAGCCGGTTTCATTT & 40 \\
\hline $7 \mathrm{X} 7 \mathrm{NC} 10$ & CGGCGGGCGGCGAAAATCCTGTTATAAATCA & 31 \\
\hline $7 \mathrm{X} 7 \mathrm{NC} 11 \mathrm{C}$ & TGCTGCGGACCGGGGGTTTCTGCCATTGTTATGTACATAA & 40 \\
\hline $7 \mathrm{X} 7 \mathrm{NC} 12$ & CTGTGCATTCAGCAAATCGTTAACCTTACAC & 31 \\
\hline $7 \mathrm{X} 7 \mathrm{NC} 13 \mathrm{C}$ & CAGTGTCACTTCGCGTCCGTGAGCTAATCATGCTTGCTTC & 40 \\
\hline $7 \mathrm{X} 7 \mathrm{NC} 14$ & ATCAGACGGTTACCTGCAGCCAGCGGGTAAAG & 32 \\
\hline $7 \mathrm{X} 7 \mathrm{NC} 15$ & AACGTGGAAAAGGGCGAAAAACCGAGCCCCCGATTTAGA & 39 \\
\hline
\end{tabular}




\begin{tabular}{|c|c|c|}
\hline $7 \mathrm{X} 7 \mathrm{NC} 16$ & GAGTCCACGGCCCTGAGAGAGTTGGGGTGGTT & 32 \\
\hline $7 \mathrm{X} 7 \mathrm{NC} 17$ & TCCAGTTGCCCACTACGTGAACCGCCGTAAAGCAC & 35 \\
\hline $7 \mathrm{X} 7 \mathrm{NC} 18$ & TAGGGTTGCTGGTTTGCCCCAGCACGGGGAGA & 32 \\
\hline $7 \mathrm{X} 7 \mathrm{NC} 19$ & AAAGAATACAGCGGGGTCATTGCTGGAGGTGTCCA & 35 \\
\hline $7 \mathrm{X} 7 \mathrm{NC} 20$ & AATCCCTTTGATGGTGGTTCCGAACTCTGTGG & 32 \\
\hline $7 \mathrm{X} 7 \mathrm{NC} 21$ & TGGTGTGAATCCGCCGGGCGCGGGTGGTGCCATCC & 35 \\
\hline $7 \mathrm{X} 7 \mathrm{NC} 22$ & TAAACATCCGGCATCAGATGCCGGATCCAGCG & 32 \\
\hline $7 \mathrm{X} 7 \mathrm{NC} 23$ & GTTTCTTTGTCACTGTTGCCCTGCCAACCGCAAGAA & 36 \\
\hline $7 \mathrm{X} 7 \mathrm{NC} 24$ & TAAATCGGAACAAAGGAAGGGAAGAAAGTACTATG & 35 \\
\hline $7 \mathrm{X} 7 \mathrm{NC} 25$ & GCATTTTTTGGGCTAGGGCGCTGGCAACCCGCCGC & 35 \\
\hline $7 \mathrm{X} 7 \mathrm{NC} 26$ & CACGCAACCAGCATCCTCATAACGGAACCTCCGGC & 35 \\
\hline $7 \mathrm{X} 7 \mathrm{NC} 27$ & TGCCAACGGCAGAACGTCAGCGTGGTGCGCGGTCC & 35 \\
\hline $7 \mathrm{X} 7 \mathrm{NC} 28$ & GGGGAAAGCCGTGCTTTC & 18 \\
\hline $7 \mathrm{X} 7 \mathrm{NC} 29$ & GTGGCGAGCCTAAAGGGTCTATCAGGGCGATGTGGAACAA & 40 \\
\hline $7 \mathrm{X} 7 \mathrm{NC} 30$ & AGCGGGCGGTCGAGGTATCACCCAAATCAAGTGCCCGAGA & 40 \\
\hline $7 \mathrm{X} 7 \mathrm{NC} 31$ & GTCACGCTCTTACGGCAGGCGCTTTCGCACTCATCGGCAA & 40 \\
\hline $7 \mathrm{X} 7 \mathrm{NC} 32$ & ACTTGTAGCACCGTCGTTGCGGTATGAGCCGGGCTCGTCA & 40 \\
\hline $7 \mathrm{X} 7 \mathrm{NC} 33$ & GTTGCTTAGGCCGATTAAAGGGAGAAGTGTT & 31 \\
\hline $7 \mathrm{X} 7 \mathrm{NC} 34$ & GCTTAATGGTTGTGTACATCGACCGCCAGCA & 31 \\
\hline $7 \mathrm{X} 7 \mathrm{NC} 35$ & CAGAGCAAAAAAAGCCGCACAGGGCGGATCA & 31 \\
\hline $7 \mathrm{X} 7 \mathrm{NC} 36$ & GTTTTTTCAGGGTAAAGTTAAACATAGCTCT & 31 \\
\hline $7 \mathrm{X} 7 \mathrm{NC} 37$ & TCCGGCAAACTGGTCTGG & 18 \\
\hline $7 \mathrm{X} 7 \mathrm{NC} 38$ & AGAATCAGAGTAAAAGAG & 18 \\
\hline $7 \mathrm{X} 7 \mathrm{NC} 39$ & TAAACAGGTGACGAGCACGTATAACGGCGAAC & 32 \\
\hline $7 \mathrm{X} 7 \mathrm{NC} 40 \mathrm{~L} 2$ & AGGAACGGCGCCGCTACAGGGCGCGCGAAAGG & 32 \\
\hline $7 \mathrm{X} 7 \mathrm{NC} 41$ & ATCCCGTAGCGCGTAACCACCACAGTGTAGCG & 32 \\
\hline $7 \mathrm{X} 7 \mathrm{NC} 42 \mathrm{R} 2$ & AGTGATGAGTCTCGTCGCTGGCAGCGTGCCGG & 32 \\
\hline $7 \mathrm{X} 7 \mathrm{NC} 43$ & TTTATAAAATACTTCTTTGATTAAACGCTCATGGA & 35 \\
\hline $7 \mathrm{X} 7 \mathrm{NC} 44$ & GTTGGGCGCCTGAGTAGAAGAACACAATATTACCG & 35 \\
\hline $7 \mathrm{X} 7 \mathrm{NC} 45$ & AACTTAAGGCGAAACGTACAGCGTCACCGGAAAC & 34 \\
\hline $7 \mathrm{X} 7 \mathrm{NC} 46$ & CACGGAAACGGAATTTGTGAGAGCAGAGGTGGAGC & 35 \\
\hline $7 \mathrm{X} 7 \mathrm{NC} 47$ & GGTGAAGGGGATGCTGAT & 18 \\
\hline $7 \mathrm{X} 7 \mathrm{NC} 48$ & CATCACGCATCAATCGTC & 18 \\
\hline $7 \mathrm{X} 7 \mathrm{NC} 49$ & GTTGTAGCTCAGTGAGGCCACCGAGCGGGAGC & 32 \\
\hline $7 \mathrm{X} 7 \mathrm{NC} 50$ & ATCACTTGTACGCCAGAATCCTGATTTTAGAC & 32 \\
\hline $7 \mathrm{X} 7 \mathrm{NC} 51$ & TCGGCCTTATTTCTGCTCATTTGCATAAAAAA & 32 \\
\hline $7 \mathrm{X7}$ NC 52 & ACCAGTCCAAGAGACGCAGAAACACGGCCTTT & 32 \\
\hline $7 \mathrm{X} 7 \mathrm{NC} 53$ & AATACCTACATAGATTCACCAGTCACAAGAGATAG & 35 \\
\hline $7 \mathrm{X} 7 \mathrm{NC} 54$ & CCAGCCATTGCATCGCTATTACGCCAGATCGGTGC & 35 \\
\hline $7 \mathrm{X} 7 \mathrm{NC} 55$ & AATCGCTGGTAAGTGCTGCAAGGCGATTCATTCAGG & 36 \\
\hline $7 \mathrm{X} 7 \mathrm{NC} 56$ & CGCCACGGGAACGGGTTTTCCCAGTCATGGTGCCG & 35 \\
\hline $7 \mathrm{X} 7 \mathrm{NC} 57$ & ACATTGGCTTTGACGCAATTAACC & 24 \\
\hline $7 \mathrm{X} 7 \mathrm{NC} 58$ & AATAAAAGACAGGAAAGTAATAAC & 24 \\
\hline
\end{tabular}




\begin{tabular}{|c|c|c|}
\hline $7 \mathrm{X7}$ NC 59 & AGGGGGATTATCCAGATCAAACTA & 24 \\
\hline $7 \mathrm{X} 7 \mathrm{NC} 60$ & TAACGCCAGGATAACCCCATGTTT & 24 \\
\hline $7 \mathrm{X} 7 \mathrm{NC} 61$ & TAAAACGACAAGCTTTATAGACTTT & 25 \\
\hline $7 \mathrm{X} 7 \mathrm{NC} 62$ & AACCCTTTTTGAATGGCTATTAGTTAACACC & 31 \\
\hline $7 \mathrm{X7}$ NC 63 & GGGCCTCTTGATAGCCCTAAAACCAGAAGAT & 31 \\
\hline $7 \mathrm{X7}$ NC 64 & CTGCGCATCGTAACCGTGCATCTGTGTAGAT & 31 \\
\hline $7 \mathrm{X} 7 \mathrm{NC} 65$ & GAAACCAGGACGACAGTATCGGCGCGGATTG & 31 \\
\hline $7 \mathrm{X7}$ NC 66 & ACAATATTCTGACCTGAAAGCGTAGATTATTT & 32 \\
\hline $7 \mathrm{X} 7 \mathrm{NC} 67$ & GCGCGAACGGACATTCTGGCCAACCGACCAGT & 32 \\
\hline $7 \mathrm{X} 7 \mathrm{NC} 68$ & TAAAAATAACTGTTGGGAAGGGCGCTGGCGAA & 32 \\
\hline $7 \mathrm{X} 7 \mathrm{NC} 69$ & GAGGGGACGCAAAGCGCCATTCGCAAGTTGGG & 32 \\
\hline $7 \mathrm{X} 7 \mathrm{NC} 70$ & GATCGCACTTTCCGGCACCGCTTCCGACGTTG & 32 \\
\hline $7 \mathrm{X} 7 \mathrm{NC} 71$ & GCCTGCAAAGCATCACCTTGCTGACTTTACAAACA & 35 \\
\hline $7 \mathrm{X} 7 \mathrm{NC} 72$ & AAAACAGACCCTCAATCAATATCAGATAATACATT & 35 \\
\hline $7 \mathrm{X} 7 \mathrm{NC} 73$ & GGGCGCACAACAGTTGAAAGGAACACTAACAACTA & 35 \\
\hline $7 \mathrm{X} 7 \mathrm{NC} 74$ & ACCGTAATCATCAACATTAAATGTCCTGTAGCCAG & 35 \\
\hline $7 \mathrm{X} 7 \mathrm{NC} 75$ & AAAATCTAACAGTGCCACGCTGAGTGGCACAG & 32 \\
\hline $7 \mathrm{X} 7 \mathrm{NC} 76$ & ATATCAAAGGTGAGGCGGTCAGTATCTTTAAT & 32 \\
\hline $7 \mathrm{X} 7 \mathrm{NC} 77$ & TGGCAAATCCGAACGAACCACCAGATCGCCAT & 32 \\
\hline $7 \mathrm{X} 7 \mathrm{NC} 78$ & GGTTATCTGGGATAGGTCACGTTGGCCAGTTT & 32 \\
\hline $7 \mathrm{X} 7 \mathrm{NC} 79$ & TAACAACCTCCGTGGGAACAAACGCTCAGGAA & 32 \\
\hline $7 \mathrm{X} 7 \mathrm{NC} 80$ & ATTCGACAACTTTATTAATTTTAAAAGATATAATC & 35 \\
\hline $7 \mathrm{X} 7 \mathrm{NC} 81$ & TGAGGATTTAGAATTTTGCGGAACAAAATTCCTGA & 35 \\
\hline $7 \mathrm{X} 7 \mathrm{NC} 82$ & ATAGATTAGAGGTTAATATTTTGTTAAATTTAAAT & 35 \\
\hline $7 \mathrm{X} 7 \mathrm{NC} 83$ & CTTTAAAATATCTTGTTAAATCAGCTCGCCCCAAA & 35 \\
\hline $7 \mathrm{X} 7 \mathrm{NC} 84$ & TAATTCGCGGAACGCCAT & 18 \\
\hline $7 \mathrm{X} 7 \mathrm{NC} 85$ & CCCGAACGCGTATTAAGCAAATGA & 24 \\
\hline $7 \mathrm{X} 7 \mathrm{NC} 86$ & ACATTATCAGTATTAGAACCTCAA & 24 \\
\hline $7 \mathrm{X} 7 \mathrm{NC} 87$ & CAGAAGGACCGTCAATTGGTCAGT & 24 \\
\hline $7 \mathrm{X} 7 \mathrm{NC} 88$ & TTAAATTTTTTAGGAGTTGAGGAA & 24 \\
\hline $7 \mathrm{X} 7 \mathrm{NC} 89$ & ACCAATAGTCTGGCCTTGAGCGAG & 24 \\
\hline $7 \mathrm{X} 7 \mathrm{NC} 90$ & CTGATTGGAAGGGTTAGAACCTAAGAGGCGA & 31 \\
\hline $7 X 7$ NC 91 & TTATCAGATGCACGTAAAACAGAATTGCTTT & 31 \\
\hline $7 \mathrm{X} 7 \mathrm{NC} 92$ & TGTAAACAGATTTTCAGGTTTAAACATCGGG & 31 \\
\hline $7 \mathrm{X} 7 \mathrm{NC} 93$ & AACAGGAAAAGAGAATCGATGAAAGAGTCTG & 31 \\
\hline $7 \mathrm{X} 7 \mathrm{NC} 94$ & TGTACCCCGAGCATGTCA & 18 \\
\hline $7 X 7$ NC 95 & TGAATAATGTTTGGATTA & 18 \\
\hline $7 \mathrm{X} 7 \mathrm{NC} 96 \mathrm{~L} 4$ & AAATTATTTGATGGCAATTCATCATTTGAGTA & 32 \\
\hline $7 \mathrm{X} 7 \mathrm{NC} 97$ & AATTGCGTGCGGAATTATCATCATGAAACCAC & 32 \\
\hline $7 \mathrm{X} 7 \mathrm{NC} 98 \mathrm{R} 4$ & GAATATACGATTGTATAAGCAAATAATTCGCA & 32 \\
\hline $7 X 7$ NC 99 & GTAAAACTGTTGATAATCAGAAAAATTTTTTA & 32 \\
\hline $7 \mathrm{X} 7 \mathrm{NC} 100 \mathrm{C}$ & ATTATTCGATGATGAAACAAACATGAGTGAGCTAACTCA & 39 \\
\hline $7 \mathrm{X} 7 \mathrm{NC} 101 \mathrm{C}$ & GAATACCAAATTACATTTAACAAAAGCATAAAGTGTAAA & 39 \\
\hline
\end{tabular}




\begin{tabular}{|c|c|c|}
\hline $7 X 7 \mathrm{NC} 102 \mathrm{C}$ & AGAAACATTTTTTAATGGAAACACCGCTCACAATTCCAC & 39 \\
\hline $7 \mathrm{X} 7 \mathrm{NC} 103 \mathrm{C}$ & GAGCAAACATGTGAGTGAATAACGTCATAGCTGTTTCCT & 39 \\
\hline $7 \mathrm{X} 7 \mathrm{NC} 104$ & ACAAAGGCTGTCGCTATT & 18 \\
\hline 7X7 NC 105 & GCAAAAGAAATTTCAATT & 18 \\
\hline 7X7 NC 106 & ACAAAATTAGTTACAAAATCGCGCCCATATCA & 32 \\
\hline 7X7 NC 107 & GAATTACCATAACGGATTCGCCTGAATAAAGA & 32 \\
\hline $7 \mathrm{X} 7 \mathrm{NC} 108$ & ATCAATATAGTAACAGTACCTTTTCGTCAGAT & 32 \\
\hline \multirow[t]{2}{*}{$7 \mathrm{X} 7 \mathrm{NC} 109$} & TGTAAATCATCAGGTCATTGCCTGCGGTAATC & 32 \\
\hline & 2 photo-cleavable guide strands & \\
\hline $7 \mathrm{X} 7 \mathrm{NC} 40 \mathrm{~L} 2$ 16nt & $\begin{array}{c}\text { AGCTGAAACGACATAC-TTTXTTT- } \\
\text { AGGAACGGCGCCGCTACAGGGCGCGCGAAAGG }\end{array}$ & \\
\hline \multirow[t]{2}{*}{$7 \mathrm{X} 7 \mathrm{NC} 96 \mathrm{~L} 416 \mathrm{nt}$} & $\begin{array}{c}\text { ATTGCAAGGAGTTTAT-TTTXTTT- } \\
\text { AAATTATTTGATGGCAATTCATCATTTGAGTA }\end{array}$ & \\
\hline & 2 capture strands & \\
\hline $7 \mathrm{X} 7 \mathrm{NC} 42 \mathrm{R} 216 \mathrm{nt}$ & $\begin{array}{c}\text { CAATCGGAATGCATGG-TTTTTTTT- } \\
\text { AGTGATGAGTCTCGTCGCTGGCAGCGTGCCGG }\end{array}$ & \\
\hline $7 \mathrm{X} 7 \mathrm{NC} 98 \mathrm{R} 4$ 16nt & $\begin{array}{l}\text { TTAAGCCTGAAGAAAT-TTTTTTTT- } \\
\text { GAATATACGATTGTATAAGCAAATAATTCGCA }\end{array}$ & \\
\hline
\end{tabular}

\section{Synthesis strategy for the single-stranded DNA containing the bcl-2 hairpin forming} sequence.

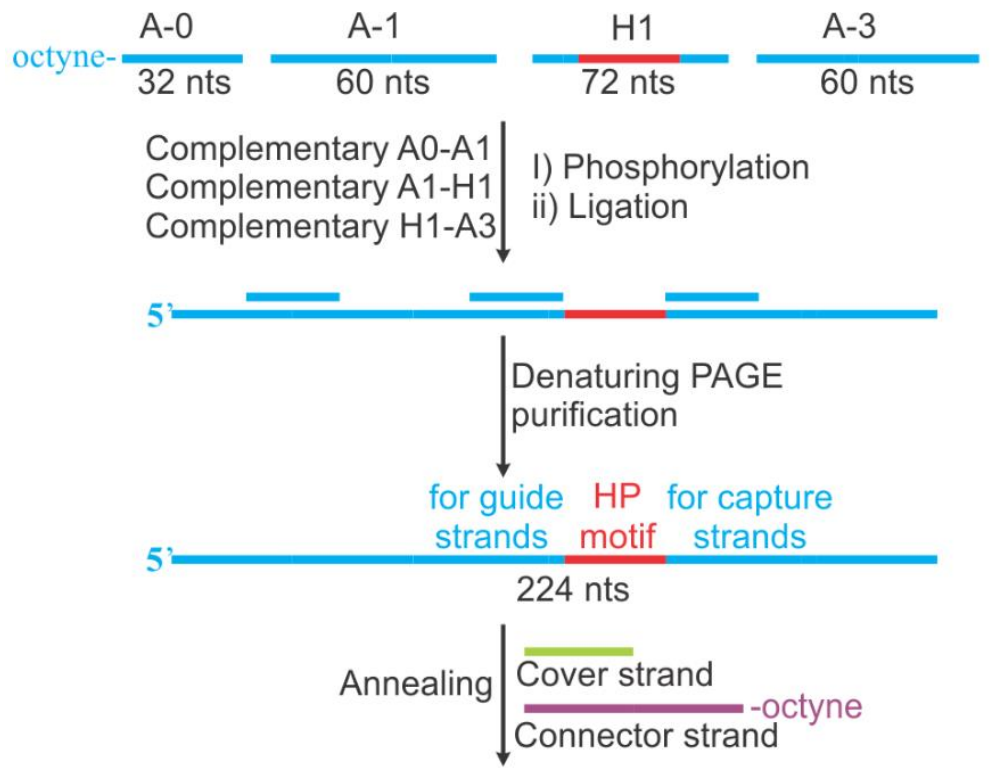

octyne-

octyne

Figure S3. Flow chart for the synthesis of the 224-nt DNA fragment containing the bcl-2 hairpin sequence. This fragment passes through the DNA nanocage. 
The following sequences of DNA oligos were used for the construct preparation.

Respective sequences are listed below.

A0: 5'-TTA CCT TTC CCC TCT G-GC AAA ACT ATT CCG GT

A1: 5'-CTT GTT GCT CAG ACT C-TA ACT CAT TGA TAC TCA TTT ATA AAC TCC TTG CAA TGT ATG TCG

H1: 5'- TTT CAG CTA AAC TTT TTC ACC ACA GCC CCG CTC CTT TTG GAG CGG GGC TGT GGT GTT TTT AAA CAT TTC TTC

A3: 5'-AGG CTT AA-C CAT GCA TTC CGA TTG CAG CTT GCA TCC ATT GCA TCG CTT GAA TTG TCC AGC

Complementary A0-A1: 5'-CTG AGC AAC AAG -ACC GGA ATA GTT

Complementary A1-H1: 5'- GTTT-AGCTGAAA-CGACATAC-ATTG

Complementary H1-A3: 5'- ATGG-TTAAGCCT-GAAGAAAT-GTTT

5'-Cover strand: 5'- GAG TAT CAA TGA GTT AGA GTC TGA GCA ACA AG

3'-Connector strand: 5'- GCC CTT TGT GGT TCA C-GT AGT GGG TGG CGC CC-G CTG GAC AAT TCA AGC-GAT GCA ATG GAT GCA A 


\section{Synthesis strategy for the single-stranded DNA that contains a telomeric G-quadruplex}

sequence and a telomeric i-motif sequence.

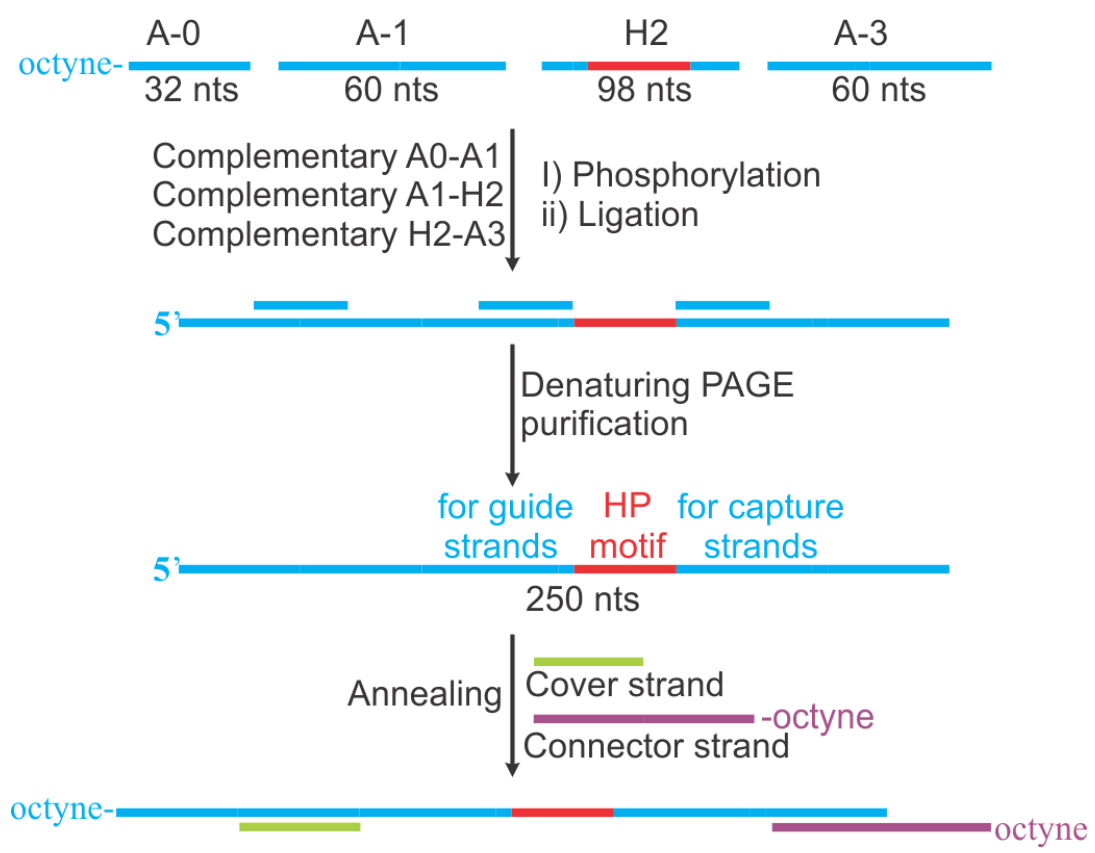

Figure S4. Flow chart for the synthesis of the 250-nt DNA fragment containing a hairpin forming sequence. A telomeric G-quadruplex sequence and a complementary $i$-motif sequence can form the stem of the hairpin (H2). This fragment passes through the DNA nanocage.

A0: 5'-TTA CCT TTC CCC TCT G-GC AAA ACT ATT CCG GT

A1: 5'-CTT GTT GCT CAG ACT C-TA ACT CAT TGA TAC TCA TTT ATA AAC TCC TTG CAA TGT ATG TCG

H2: 5'-TTT CAG CTA AAC TTT TTGT TAG GGT TAG GGT TAG GGT TAG GGT TAG GTT TTC CTA ACC CTA ACC CTA ACC CTA ACC CTA ACT TTT T AAA CAT TTC TTC

A3: 5'-AGG CTT AA-C CAT GCA TTC CGA TTG CAG CTT GCA TCC ATT GCA TCG CTT GAA TTG TCC AGC

Complementary A0-A1: 5'-CTG AGC AAC AAG -ACC GGA ATA GTT

Complementary A1-H2: 5'- GTTT-AGCTGAAA-CGACATAC-ATTG

Complementary H2-A3: 5'- ATGG-TTAAGCCT-GAAGAAAT-GTTT 
5'-Cover strand: 5'- GAG TAT CAA TGA GTT AGA GTC TGA GCA ACA AG

3'-Connector strand: 5'- GCC CTT TGT GGT TCA C-GT AGT GGG TGG CGC CC-G CTG GAC AAT TCA AGC -GAT GCA ATG GAT GCA A

4. Characterization of the single molecular DNA nanocage construct by AFM.

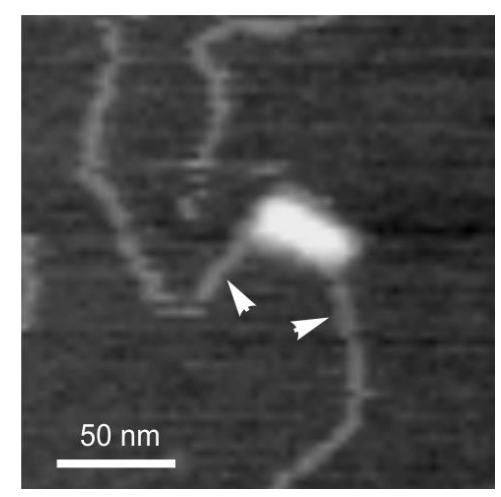

Figure S5. AFM image of a $15 \mathrm{~nm} \times 15 \mathrm{~nm}$ DNA nanocage with the hairpin that contains tetraplex (G-quadruplex and i-motif) forming sequences in its complementary stem strands. The hairpin sequences are attached to dsDNA handles (arrowheads). 


\section{Unfolding/refolding force and change-in-contour-length measurements.}

The unfolding of hairpin was marked by a sudden drop in force with a change in the extension of the tether which was recorded in F-X curves. The unfolding and refolding forces were measured from these F-X curves.

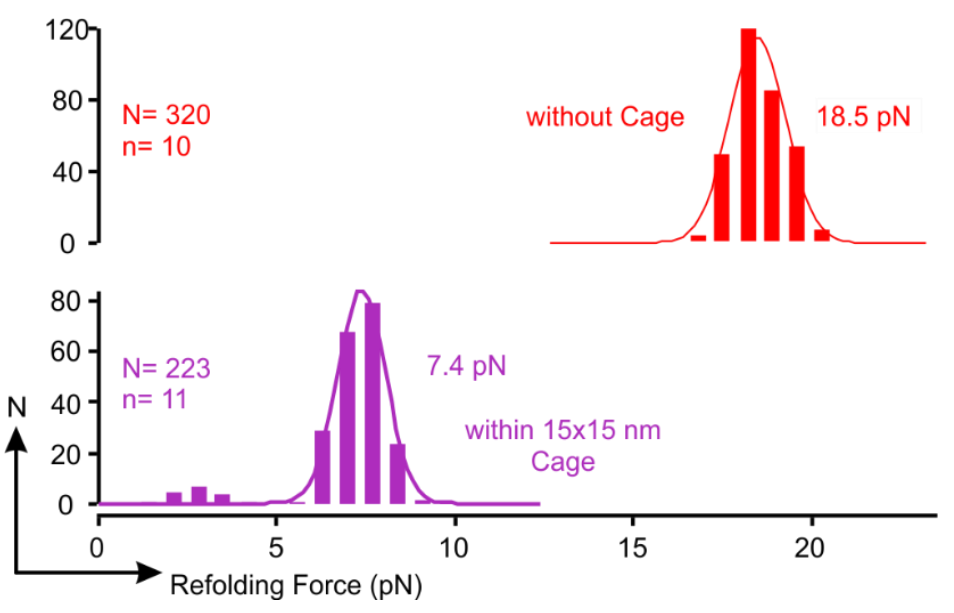

Figure S6. Refolding force histograms of the bcl-2 hairpin without (red) and within the $15 \times 15$ $\mathrm{nm}$ nanocage (purple) in a $20 \mathrm{mM}$ Tris buffer (pH 7.8) supplemented with $10 \mathrm{mM} \mathrm{MgCl}, 1 \mathrm{mM}$ EDTA, and $100 \mathrm{mM} \mathrm{KCl}$ at $25^{\circ} \mathrm{C} . \mathrm{N}$ and $\mathrm{n}$ represent the numbers of unfolding features and molecules, respectively. Refolding force was measured as the force at which the last refolding transition was observed during rapid unfolding and refolding transitions (see insets of Figure $2 b \& c$ ).

Change-in-extension $(\Delta x)$ was obtained by measuring the extension change between the two data points flanking the unfolding and refolding events at the same force $(F)$. Change-incontour-length $(\Delta \mathrm{L})$ was obtained from the $\Delta x$ using modified worm-like chain model (Eqn S1). ${ }^{\text {S2- }}$ 3

$$
\frac{\Delta x}{\Delta L}=1-\frac{1}{2}\left(\frac{k_{b} T}{F P}\right)^{1 / 2}+\frac{F}{S} \ldots \ldots \ldots \text { Eqn } S 1
$$

where $k_{\mathrm{b}}$ is the Boltzmann constant, $T$ is absolute temperature, $P$ is the persistent length $(50.9 \pm$ $1.6 \mathrm{~nm})$ and $S$ is the stretching modulus $(1168 \pm 119 \mathrm{pN})$ of the duplex DNA handles. ${ }^{\mathrm{S}}$ 


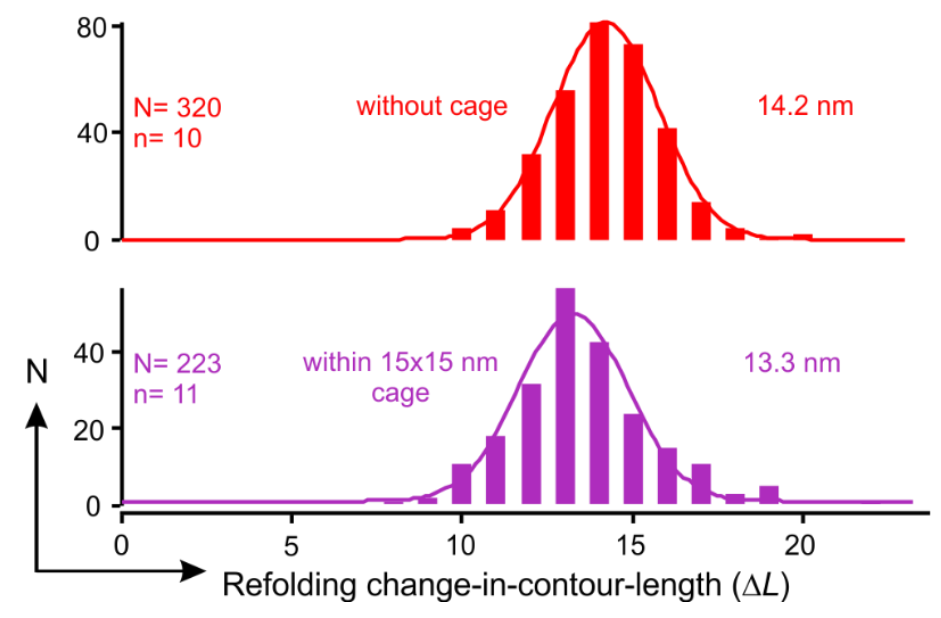

Figure S7. Refolding change-in-contour-length histogram of the bcl-2 hairpin without (red) and within (purple) the $15 \mathrm{~nm} \times 15 \mathrm{~nm}$ nanocage in a $20 \mathrm{mM}$ Tris buffer $(\mathrm{pH}=7.8)$ supplemented with $10 \mathrm{mM} \mathrm{MgCl}, 1 \mathrm{mM} \mathrm{EDTA}$, and $100 \mathrm{mM} \mathrm{KCl}$ at $25{ }^{\circ} \mathrm{C} . \mathrm{N}$ and $n$ represent the numbers of unfolding features and molecules, respectively. 

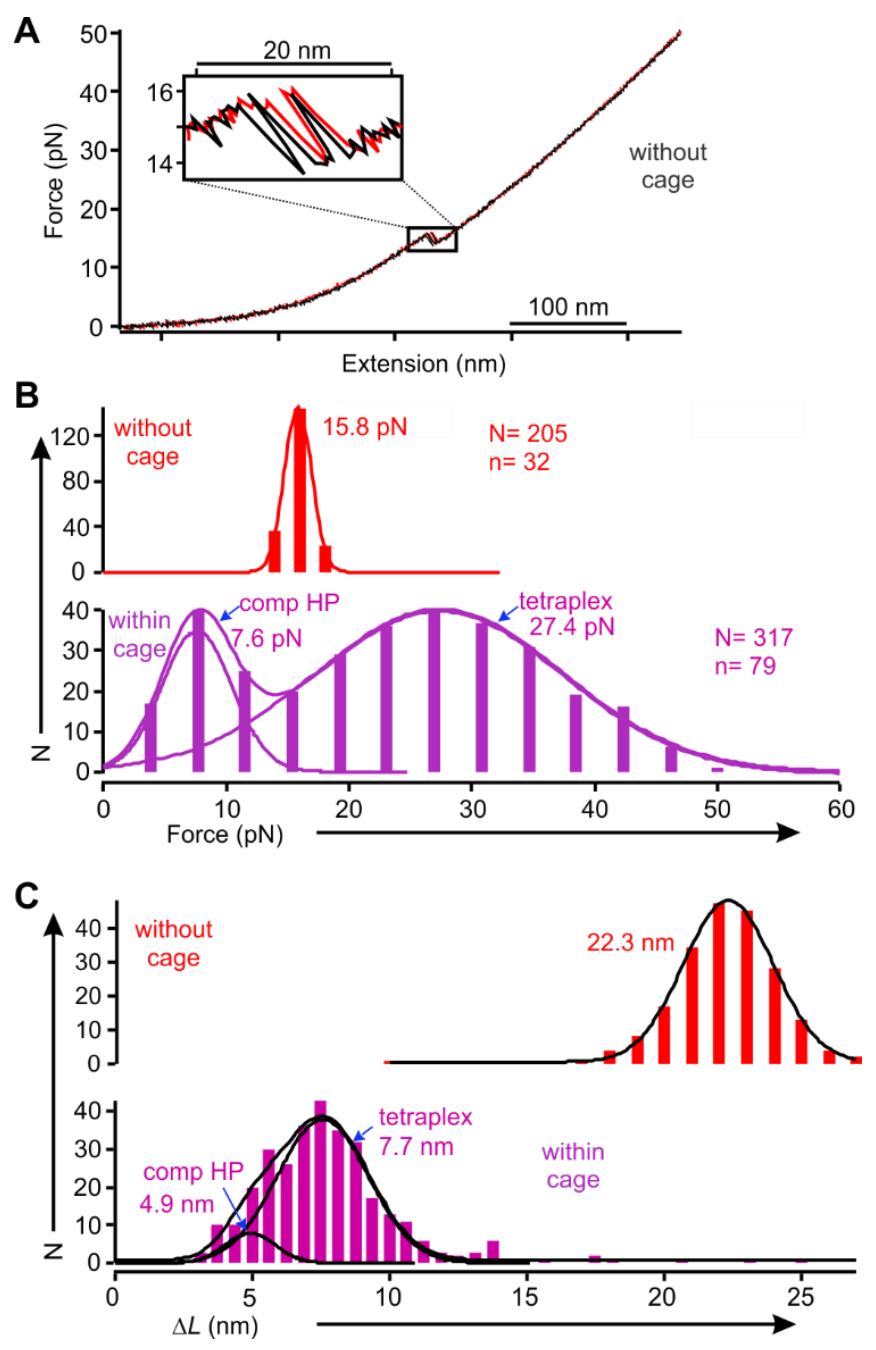

Figure S8. (A) A typical force versus extension curve of the DNA hairpin with telomeric $G$ quadruplex and i-motifforming sequences in the complementary stem strands without nanocage. Red and black represent stretching and relaxing traces, respectively. (B) Unfolding force histograms of the DNA hairpin outside (red) and inside the $15 \times 15 \mathrm{~nm}$ nanocage (purple). Unfolding force was measured as the force at which the last unfolding transition was observed during rapid unfolding and refolding transitions (see inset of Figure S8 A). Populations of compromised hairpin structures (comp HP) and tetraplexes are indicated by arrows. Solid curves represent Gaussian fittings. $N$ and $n$ represent the numbers of unfolding features and molecules, respectively. (C) Change-in-contour-length $(\Delta L)$ histograms of the DNA hairpin outside (red) and inside (purple) the $15 \times 15 \mathrm{~nm}$ nanocage. Populations of compromised hairpin structures (comp HP) and tetraplexes are indicated by arrows. Solid curves represent Gaussian fittings. A $10 \mathrm{mM}$ 
MES (2-(N-morpholino)ethanesulfonic acid) buffer supplemented with $100 \mathrm{mM} \mathrm{KCl,} 10 \mathrm{mM}$ $\mathrm{MgCl}_{2}$, and $1 \mathrm{mM} \mathrm{EDTA}$ ( $\mathrm{pH} 5.5$ ) was used in these experiments. 


\section{Percentage populations of the hairpin and tetraplex structures.}

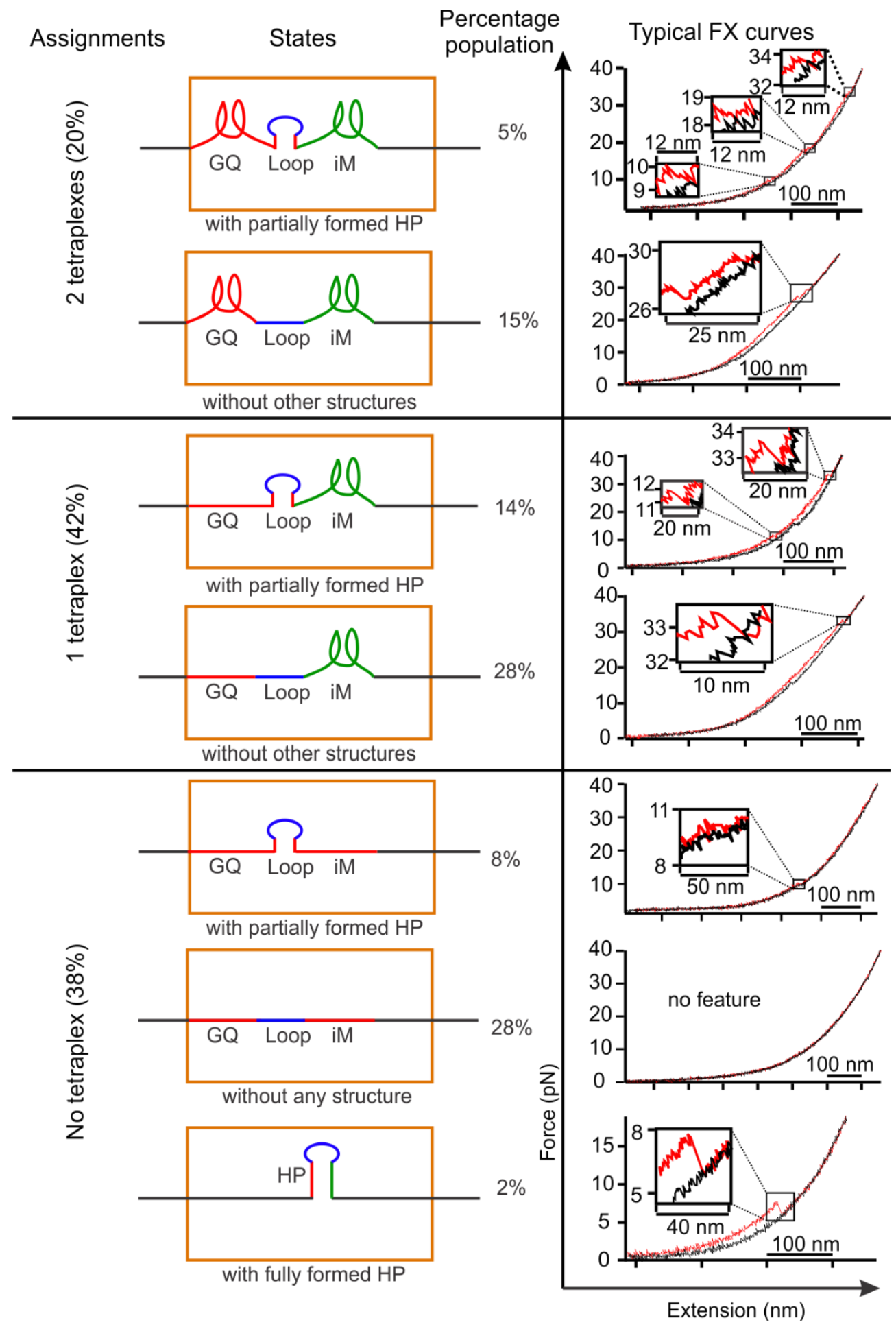

Figure S9. Percentage population of different species formed in the hairpin that contains the tetraplex forming sequences in its complementary stem strands. The associated typical force versus extension are shown to the right. 


\section{Unfolding force versus extension plots.}

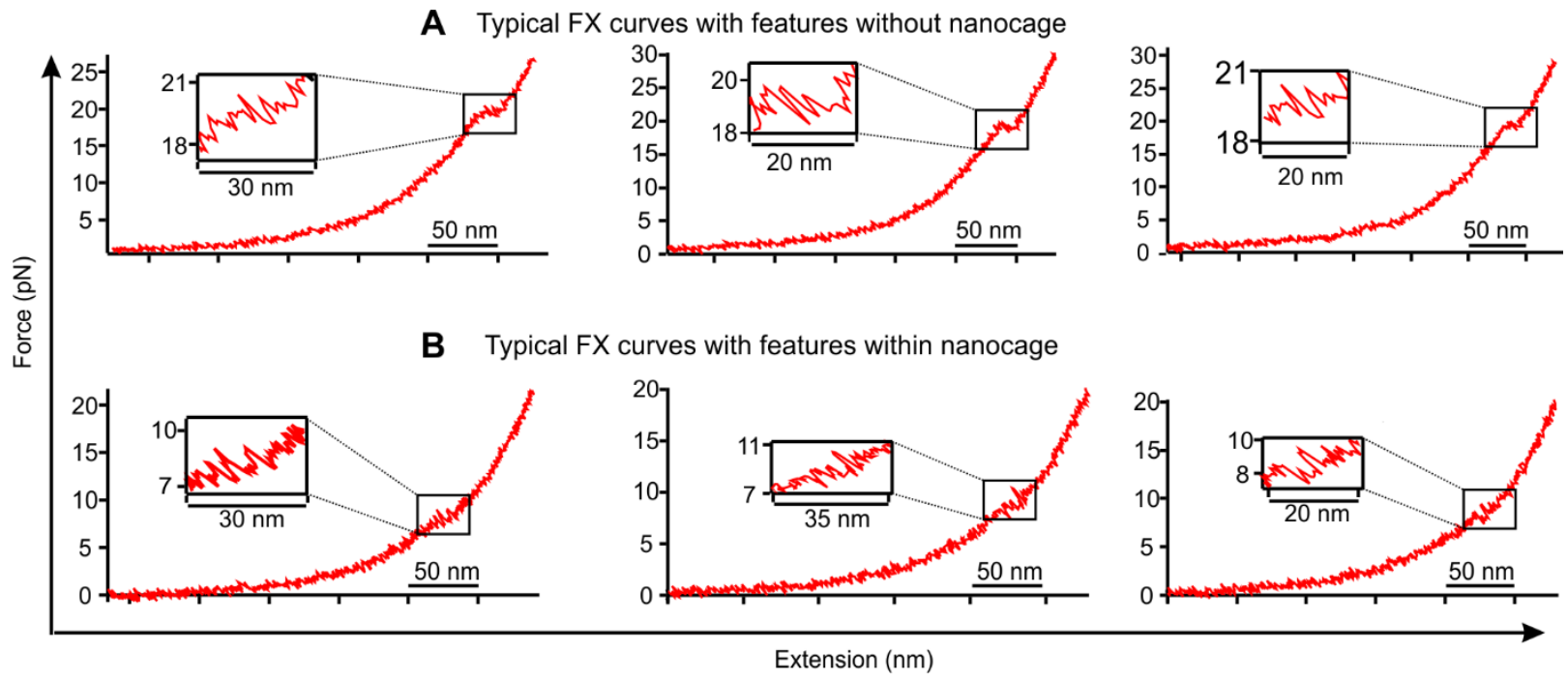

Figure S10. Typical unfolding force versus extension without A) and within B) the $15 \times 15 \mathrm{~nm}$ nanocage for bcl-2 hairpin. The features in corresponding FX curves are expanded inside insets. 


\section{A Typical unfolding FX curves without nanocage}

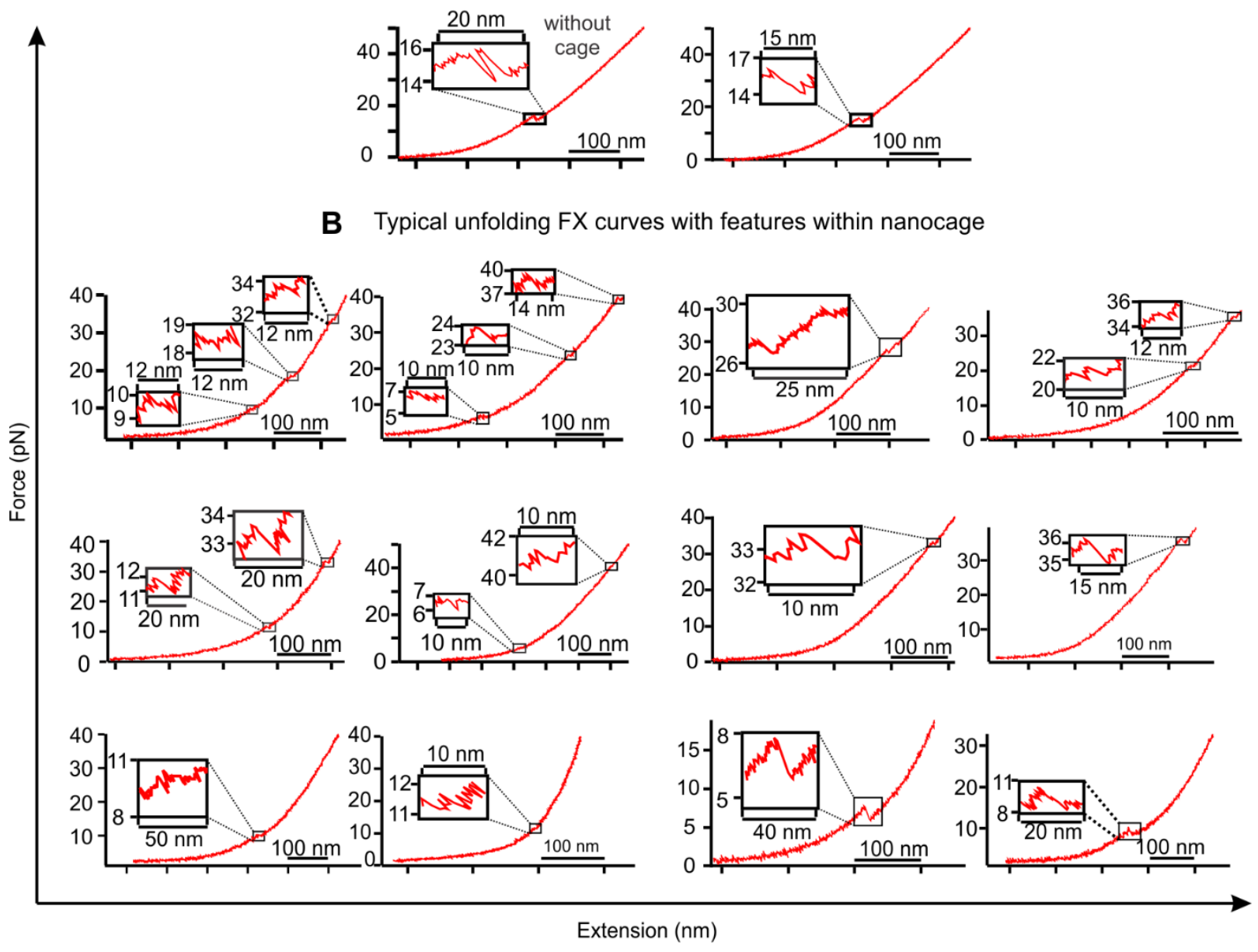

Figure S11. Typical unfolding force versus extension with transition features without A) and within $B)$ the $15 \times 15 \mathrm{~nm}$ nanocage for the hairpin with tetraplex forming sequence in the stem. The features in corresponding FX curves are expanded inside insets. 


\section{Expected change-in-contour-length $(\Delta L)$.}

The expected change-in-contour-length $(\Delta L)$ during the unfolding of the structure was calculated by the equation $\mathrm{S} 2$,

$$
\Delta L=N \times L_{\mathrm{nt}}-x \ldots \ldots \ldots \ldots \ldots \ldots(\text { Eqn S2})
$$

where $N$ is the number of nucleotides involved in the structure, $L_{\mathrm{nt}}$ is the contour length of each nucleotide $(0.43 \mathrm{~nm})^{\mathrm{S} 5-6}$, and $x$ is the end-to-end distance of the hairpin structure $\left(2 \mathrm{~nm}{ }^{\mathrm{S} 7}\right)$.

Thirty eight nucleotides (nts) (5'- CAC CAC AGC CCC GCT CCT TTT GGA GCG GGG CTG TGG TG) are involved in the formation of bcl-2 hairpin. Hence, the expected $\Delta L$ for the unfolding of the bcl-2 hairpin is $\Delta L=38 \times 0.43 \mathrm{~nm}-2 \mathrm{~nm}=14.3 \mathrm{~nm}$.

Similarly, 64 nts (5'-GTT AGG GTT AGG GTT AGG GTT AGG GTT AGG TTT TCC TAA CCC TAA CCC TAA CCC TAA CCC TAA C) are involved in the formation of the hairpin that contains tetraplexes forming sequences in its stem. Hence, the expected $\Delta L$ for the unfolding of the tetraplex containing hairpin is $\Delta L=64 \times 0.43 \mathrm{~nm}-2 \mathrm{~nm}=25.5 \mathrm{~nm}$.

\section{Calculation of the change in free energy of unfolding $\left(\Delta G_{\text {unfold }}\right)$.}

The change in free energy of unfolding was calculated from the corresponding works from unfolding events using Jarzynski's equality equation (Eqn S3). ${ }^{\text {S8 }}$

$$
\Delta G_{\text {unfold }}=-k_{B} \operatorname{Tln} \sum_{i=1}^{N} \frac{1}{N} \exp \left(-\frac{W_{i}}{k_{B} T}\right) \ldots \ldots \ldots . .(\text { Eqn S3 })
$$

where $k_{B}$ is the Boltzmann constant, $T$ is the absolute temperature, $N$ is the number of repetitions of the experiments, and $W$ is the non-equilibrium work done to unfold the bcl-2 hairpin. Work done was calculated using the equation,

$$
W_{i}=\sum_{i=1}^{N} F_{i} \Delta x_{i} \ldots \ldots \ldots(\text { Eqn } \mathrm{S} 4)
$$


here $F$ and $\Delta x$ are the unfolding force and the change in extension due to mechanical unfolding of the structure, respectively.

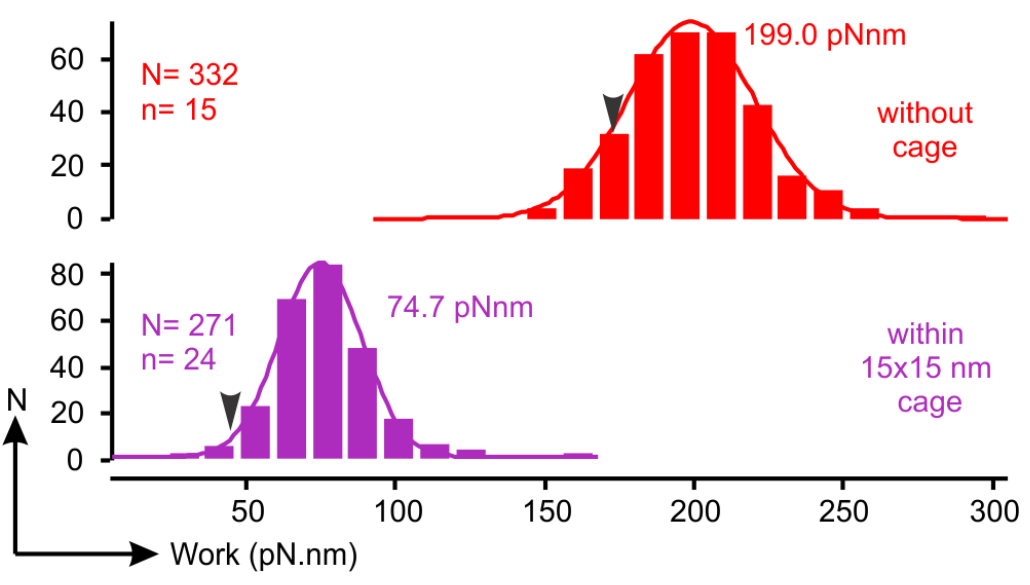

Figure S12. Unfolding work histograms of the bcl-2 hairpin without (red) and within (purple)

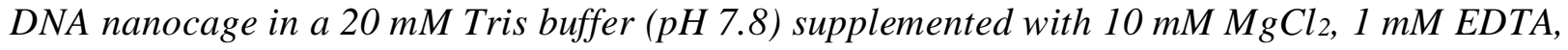
and $100 \mathrm{mM} \mathrm{KCl}$ at $25^{\circ} \mathrm{C}$. The arrowheads indicate the values of the change in the free energy of unfolding respective hairpins. $N$ and $n$ represent the numbers of unfolding features and molecules, respectively. 
10. Estimation of the change in free energy of hairpin unfolding using mfold ${ }^{\circledR}$.
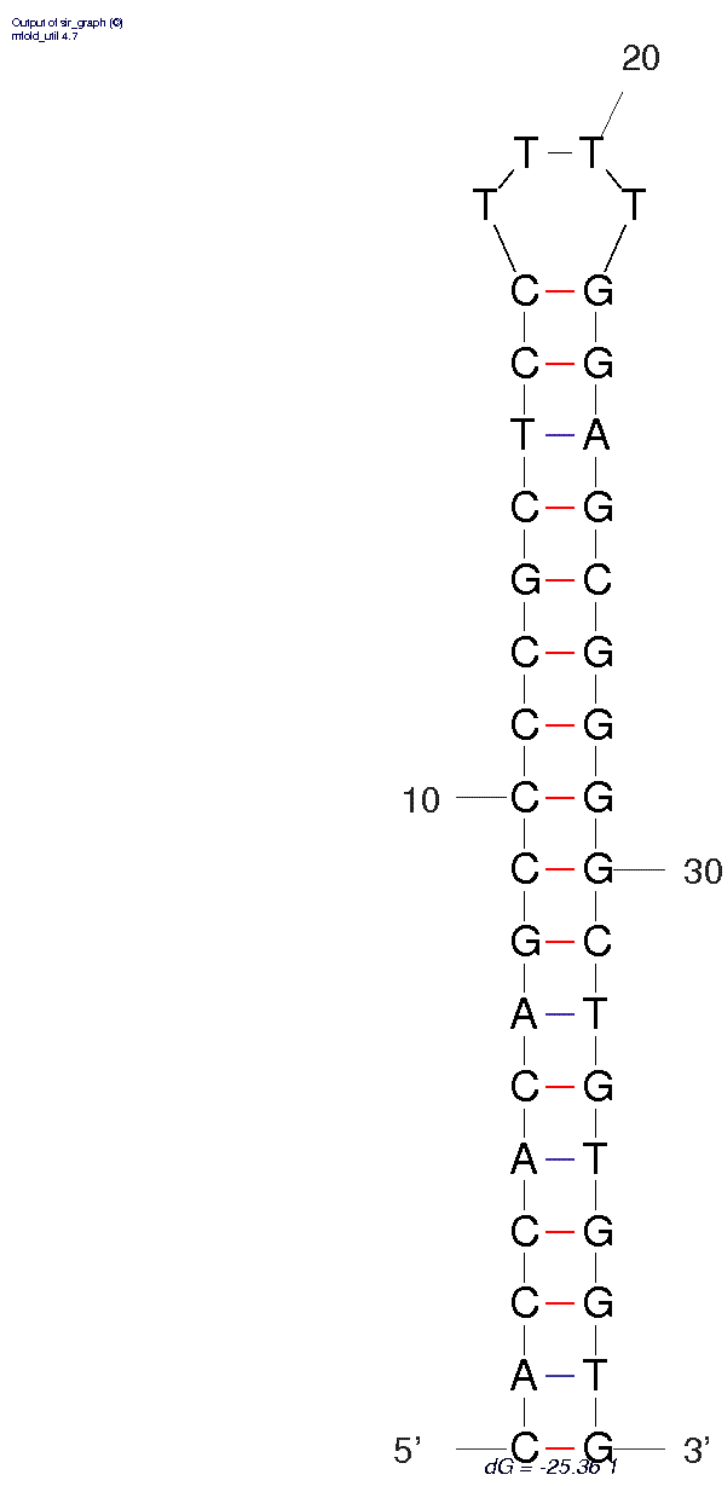

Figure S13. Bcl-2 hairpin structure generated from the mfold ${ }^{\circledR} .{ }^{S 9}$ The change in free energy of unfolding was found to be $-25.36 \mathrm{kcal}$ in $100 \mathrm{mM} \mathrm{Na}+$ at $25{ }^{\circ} \mathrm{C}$. 


\section{Energy stored in the unfolding pathway of the bcl-2 hairpin.}

The free energy profile during the unfolding pathway $(L)$ of the bcl-2 hairpin at 0 force $\left(G(\mathrm{~L}, \mathrm{~F}=0)_{\mathrm{Hp}}\right)$ can be obtained by correcting the free energy change at specific unfolding force $\left(G(\mathrm{~L}, \mathrm{~F})_{\mathrm{Hp}}\right)$ with the energies contributed by the optical trap energy $(G(\mathrm{~F})$ trap $)$, extension of duplex DNA handles from $0 \rightarrow \mathrm{F}(G(\mathrm{~F}) \mathrm{dsDNA})$, extension of unfolded part of the hairpin from $0 \rightarrow \mathrm{F}(G(\mathrm{~L}$, $\left.\mathrm{F})_{\mathrm{unfold}-\mathrm{Hp}}\right)$, and extension of folded part of the hairpin from $0 \rightarrow \mathrm{F}\left(G(\mathrm{~L}, \mathrm{~F})_{\text {fold-Hp }}\right) .{ }^{\mathrm{S} 10}$ The correction can be expressed in the equation (eqn S5) below.

$$
G(\mathrm{~L}, \mathrm{~F}=0)_{\mathrm{Hp}}=G(\mathrm{~L}, \mathrm{~F})_{\mathrm{Hp}}-G(\mathrm{~F})_{\text {trap }}-G(\mathrm{~F})_{\text {dsDNA }}-G(\mathrm{~L}, \mathrm{~F})_{\text {unfold-Hp }}-G(\mathrm{~L}, \mathrm{~F})_{\text {fold }-\mathrm{Hp}} \ldots \ldots . .(\text { Eqn S5) }
$$

\section{Point spread function used to convert species populations to relative free energies.}

Point Spread Function (PSF) was obtained from a control construct (construct without the hairpin forming sequence). PSF is defined as the Gaussian width of the change-in-contour-length histogram of the control construct at specific force range of interest (i.e., unfolding forces of the bcl-2 hairpins within and without nanocages). The obtained PSF value was used in the changein-contour-length histogram of the bcl-2 hairpin construct. The PSF values at different force are shown in each histogram.
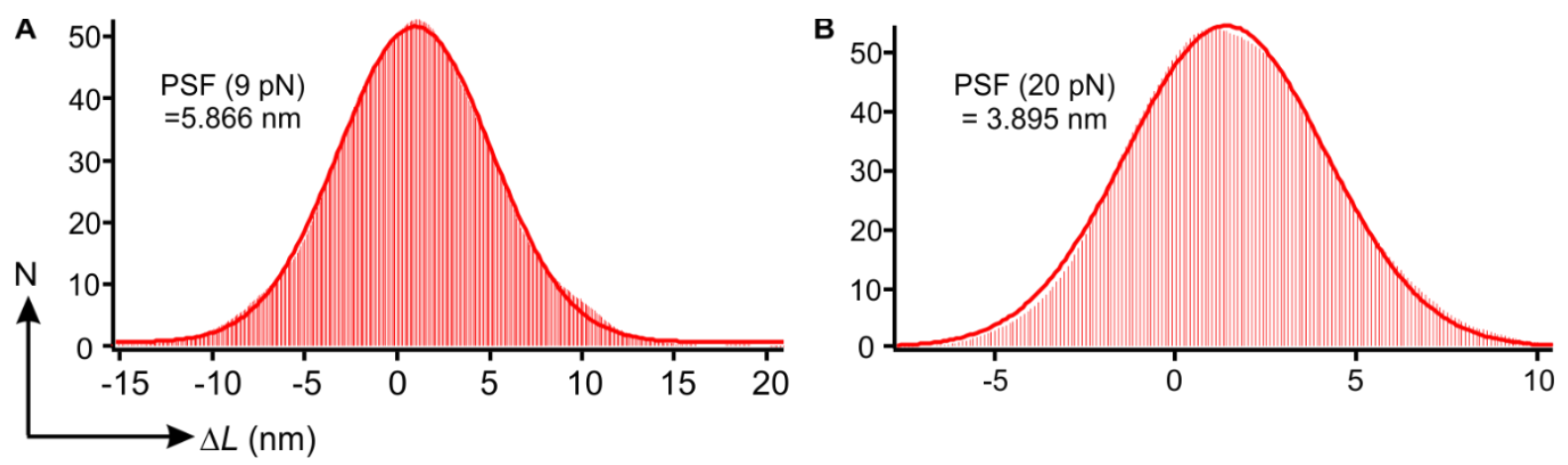

Figure S14. Change-in-contour-length ( $\Delta L)$ histograms of the control construct (construct without hairpin forming sequence) at (A) $9 \mathrm{pN}(P S F=5.866 \mathrm{~nm})$ and (B) $20 \mathrm{pN}(P S F=3.895$ $\mathrm{nm})$. The $\Delta L$ was calculated from the change-in-extension between the stretching and relaxing force-extension curves at the same force. Notice there is no feature involved in this construct. 


\section{Coarse-grained molecular dynamics (MD) simulation of bcl-2 hairpin inside the nanocages.}

The simulation was done at $277 \mathrm{~K}$. We placed the folded bcl-2 DNA hairpin (previously simulated for $300 \mu$ s) inside a 9×9 nm origami nanocage (obtained from Cadnano) in the oxDNA2 platform. ${ }^{\text {S11 }}$ The DNA hairpin contains a $25-n t$ ssDNA overhang on the guide strand end (Figure S1) and a 9-nt ssDNA with a 16-bp dsDNA on the other end that is connected to the origami nanocage. Energy minimization of the structure was performed for 30 ns. Finally, the coarsegrained molecular dynamic simulation was performed for $7.5 \mu \mathrm{s}$ at $277 \mathrm{~K}$. Plot of the distance between the end of the ssDNA overhang of the hairpin and the opposite end of the nanocage versus simulation steps (Figure S15 C) shows a stable oscillating motion. Similarly, molecular simulations were performed for the folded bcl-2 DNA hairpin (Figure S15 D) and the folded DNA hairpin with tetraplex forming sequences in its complementary stem strands (Figure S15 E) inside the $15 \times 15 \mathrm{~nm}$ origami nanocage.
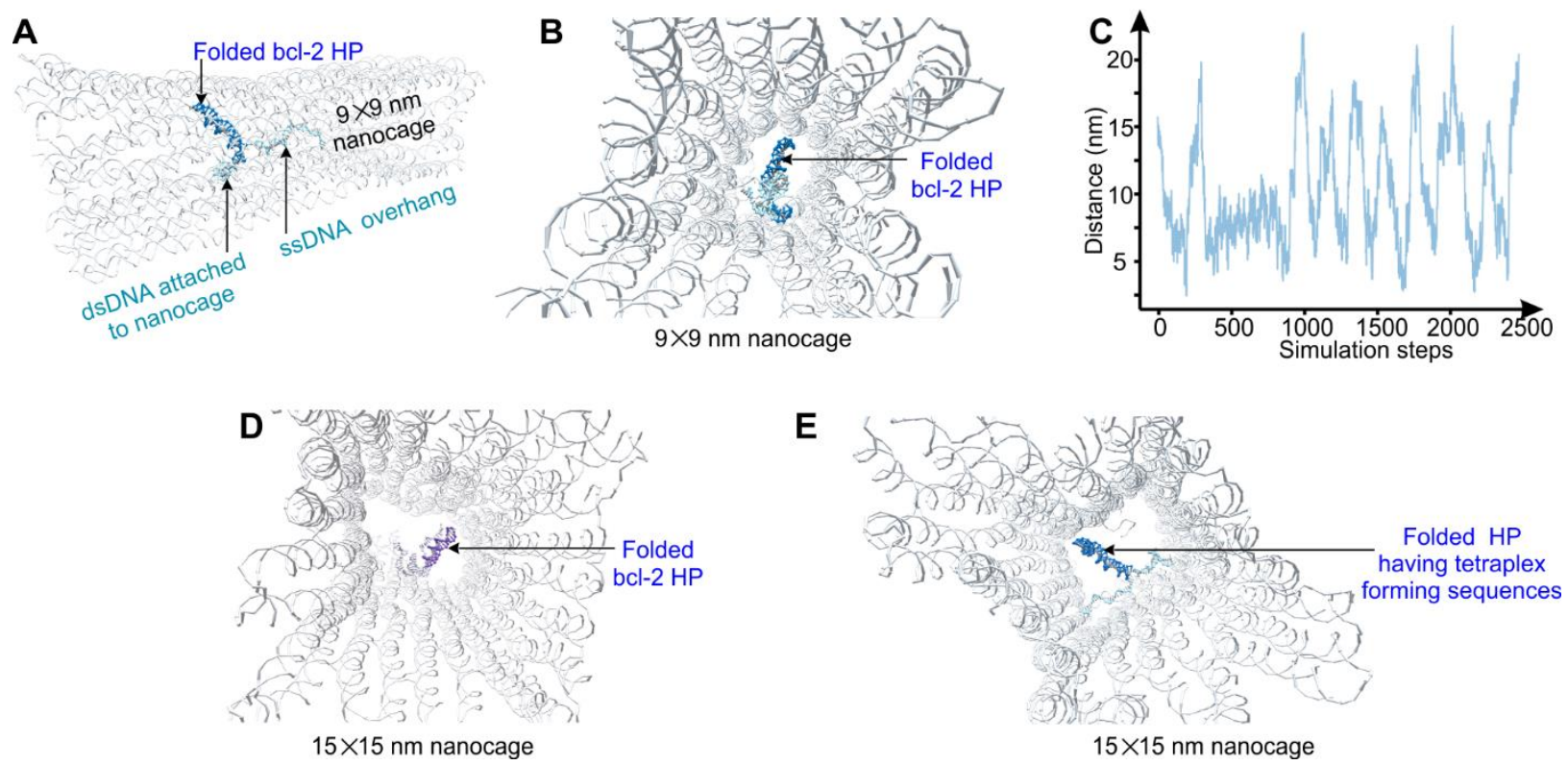

Figure S15. Representations of the coarse-grained MD simulation models of folded hairpins (blue) inside DNA origami nanocages (grey). A) Front view and B) side view of the folded bcl-2 hairpin (blue) inside the $9 \times 9 \mathrm{~nm}$ DNA origami nanocage (grey). C) Plot of the distance between the end of the 25-nt ssDNA overhang attached to the bcl-2 hairpin and the opposite end of the nanocage versus simulation steps. This plot shows a stable oscillating pattern. D) A folded bcl-2 
hairpin and E) a folded hairpin with tetraplex forming sequences in its complementary stem strands inside the $15 \times 15 \mathrm{~nm}$ DNA origami nanocage.

\section{Quantitation of factors in the reduced stability of the duplex DNA.}
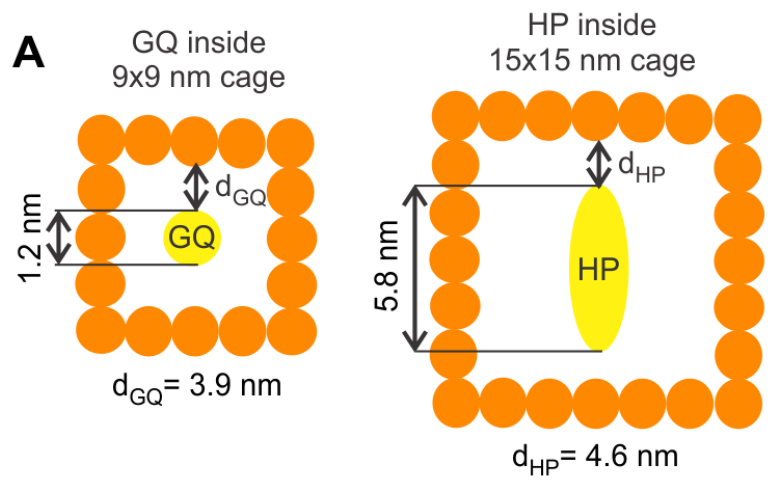

B

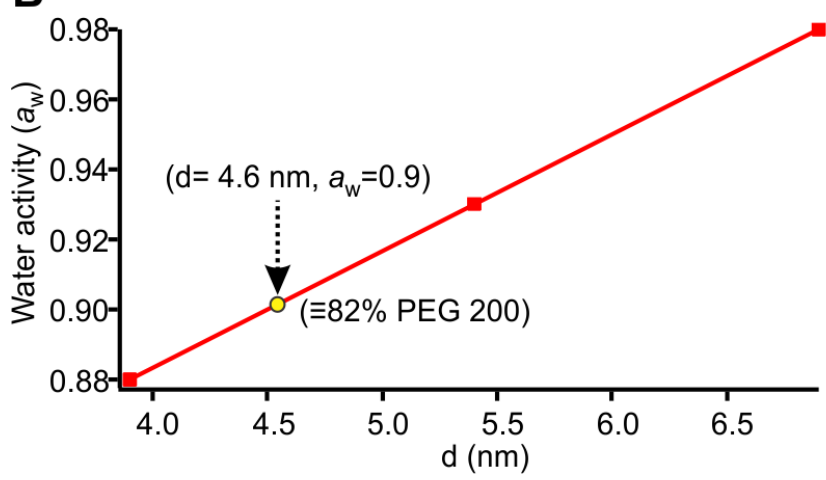

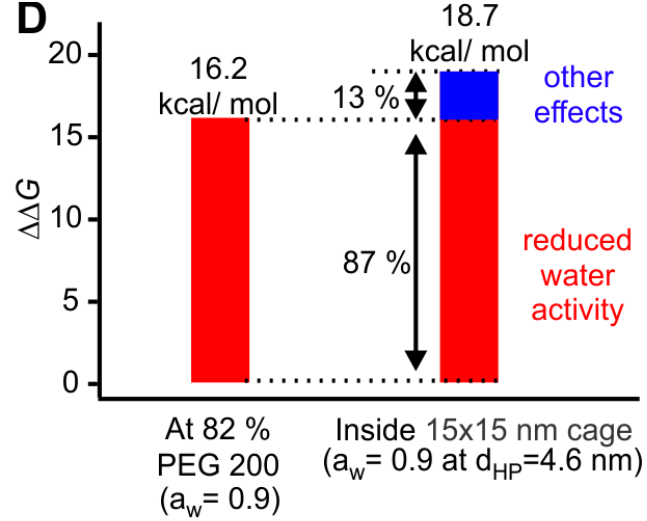

C

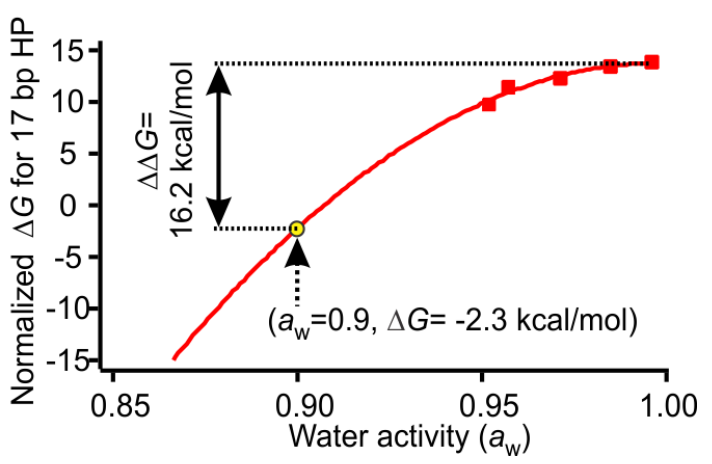

Figure S16. Quantitation of the effect of water activity on the stability of the bcl-2 hairpin inside the nanocage. A) Schematic of the G-quadruplex (GQ) inside the $9 \times 9 \mathrm{~nm}$ nanocage (left) and the bcl-2 hairpin (HP) inside the $15 \times 15 \mathrm{~nm}$ nanocage (right). " $d$ " indicates the closest distance between the DNA species (GQ or HP) and the DNA nanocage wall. B) Plot of water activity $\left(a_{w}\right)$ versus $d$ (distance between the base of the stem of the bcl-2 hairpin and the cage wall). Water activity of 0.9 was found by interpolation at $d=4.6 \mathrm{~nm}$. C) Plot of the normalized change in the unfolding free energy for a 17-bp hairpin versus water activity $\left(a_{w}\right)$. The plot is fitted with a threeterm polynomial function which is extrapolated to find the change in the unfolding free energy at water activity $\left(a_{w}\right)$ of 0.9. D) Comparative plots of the difference in the unfolding free energy change $(\Delta \Delta G)$ for a 17-bp hairpin in 82\% equivalent PEG 200 and that inside the $15 \times 15 \mathrm{~nm}$ 
nanocage. $\Delta \Delta G$ here is calculated against the free energy change of unfolding the 17-bp hairpin at water activity $a_{w}=1$.

Water activity inside the nanocage is a function of the distance from the nanocage wall. Inside the nanocage, the water activity in the middle of the nanocage is the highest while the vicinity of the nanocage wall has the lowest water activity due to increased ion-dipole interactions. The water activity $\left(a_{\mathrm{w}}\right)$ at the center of the $15 \times 15 \mathrm{~nm}$ nanocage was reported to be 0.98 which was measured based on the G-quadruplex (GQ) inside the nanocage. ${ }^{\mathrm{S}} 4 \mathrm{The}$ GQ has a spherical shape with a diameter of $\sim 1.2 \mathrm{~nm}$ and is confined at the center of the nanocage (Figure S16 A, left). On the other hand, molecular simulation of 17-bp (base pair) bcl-2 hairpin inside the $9 \times 9 \mathrm{~nm}$ nanocage revealed that the hairpin is pointed towards the wall of the nanocage (Figure S15 A\&B) which has the lowest water activity compared to the water activity at the center of the nanocage. The bcl-2 hairpin has an elongated shape with the height of $\sim 5.8 \mathrm{~nm}(0.34 \mathrm{~nm} / \mathrm{bp} \times 17$ $\mathrm{bp}=5.8 \mathrm{~nm}$, see Figure S16 A, right). Using the Eqn S6 shown below, we found that the distance between the base of the stem of the bcl-2 hairpin and the wall of the $15 \times 15 \mathrm{~nm}$ nanocage is 4.6 $\mathrm{nm}$, which is closest to the distance between the edge of the GQ and the wall of the $9 \times 9 \mathrm{~nm}$ nanocage $(3.9 \mathrm{~nm})($ Table $\mathrm{S} 3)$.

$$
d=\frac{(\text { cage height }- \text { moelcule height })}{2} \ldots \ldots . . . .(\text { Eqn S6) }
$$

where " $d$ " is the closest distance between the edge of the DNA species (GQ or hairpin) and the wall of a nanocage.

Therefore, the water activity experienced by the bcl- 2 hairpin inside the $15 \times 15 \mathrm{~nm}$ nanocage will be close to the water activity experienced by the GQ inside the $9 \times 9 \mathrm{~nm}$ nanocage. It should be noted that the water activity at the base of the stem of the hairpin is critical since the mechanical unfolding starts from the base of the stem of the hairpin. Using the plot of water activity versus distance between the hairpin and the nanocage, the water activity at $4.6 \mathrm{~nm}$ away from the nanocage has been interpolated to be 0.90 (see Figure S16 B). The interpolated water activity $\left(a_{\mathrm{w}}=0.90\right)$ is equivalent to $82 \%$ of PEG 200 (Figure S16 B). 
Table S3. Table representing the closest distance between the DNA species (GQ or HP) and the wall of different sized DNA nanocages. $d_{G Q}$ and $d_{H P}$ indicate the distances from the corresponding nanocage wall to $G Q$ (G-quadruplex) or HP (hairpin) respectively. Water activity $\left(a_{w}\right)$ at the corresponding " $d$ " is presented inside the bracket.

\begin{tabular}{|c|c|c|}
\hline Cage size & $\boldsymbol{d}_{G Q}\left(\boldsymbol{a}_{w}\right)$ & $\boldsymbol{d H P}_{\boldsymbol{H}}\left(\boldsymbol{a}_{w}\right)$ \\
\hline $\mathbf{1 5} \times \mathbf{1 5} \mathbf{~} \mathbf{m}$ & $6.9 \mathrm{~nm}(0.98)$ & $4.6 \mathrm{~nm}(0.90)$ \\
\hline $\mathbf{1 2 \times 1 2} \mathbf{n m}$ & $5.4 \mathrm{~nm}(0.93)$ & ---- \\
\hline $\mathbf{9} \times \mathbf{9} \mathbf{n m}$ & $3.9 \mathrm{~nm}(0.88)$ & --- \\
\hline
\end{tabular}

Next, the reported changes in free energy of the 12-bp model hairpin ${ }^{\mathrm{S} 12}$ were normalized to estimate the changes in free energy for the 17-bp hairpin using Eqn S7, which were then plotted against water activities associated with different PEG 200 concentrations (Figure S16 C).

$$
\Delta G(17 \text { bp hairpin })=\frac{-\Delta G(12 \text { bp hairpin })}{12} \times 17 \ldots \ldots \ldots \ldots \ldots \ldots(\text { Eqn } \mathrm{S} 7)
$$

The plot was fitted with a three-term polynomial function which was then extrapolated to obtain the change in free energy of $-2.3 \mathrm{kcal} / \mathrm{mol}$ for $17-\mathrm{bp}$ hairpin at water activity $\left(a_{\mathrm{w}}\right)$ of 0.9 . The difference in the unfolding free energy change $(\Delta \Delta G)$ of the 17-bp hairpin between the water activity $a_{\mathrm{w}}=0.9$ and $a_{\mathrm{w}}=1(13.9 \mathrm{kcal} / \mathrm{mol})$ was calculated to be $16.2 \mathrm{kcal} / \mathrm{mol}(13.9-(-2.3)=16.2)$. For the bcl-2 hairpin on the other hand, the measured difference of the change in the free energy of unfolding with and without $15 \times 15 \mathrm{~nm}$ nanocage is $18.7 \mathrm{kcal} / \mathrm{mol}$. Considering the fact that the base of the hairpin stem is closer to the wall of the DNA nanocage, the reduced water activity will account for at least $87 \%$ reduction in the stability of the hairpin (Figure S16 D). The remaining reduced stability effect $(<13 \%)$ can be ascribed to other effects such as reduced cation activity and repulsive force between hairpin and nanocage wall. 


\section{Supplemental references.}

S1. Shrestha, P.; Jonchhe, S.; Emura, T.; Hidaka, K.; Endo, M.; Sugiyama, H.; Mao, H., Confined space facilitates G-quadruplex formation. Nat. Nanotechnol. 2017, 12 (6), 582588.

S2. Yu, Z.; Mao, H., Non-B DNA structures show diverse conformations and complex transition kinetics comparable to RNA or proteins - a perspective from mechanical unfolding and refolding experiments. Chem. Rec. 2013, 13, 102-116.

S3. Baumann, C. G.; Smith, S. B.; Bloomfield, V. A.; Bustamante, C., Ionic effects on the elasticity of single DNA molecules. Proc. Natl. Acad. Sci. USA. 1997, 94, 6185-6190.

S4. Jonchhe, S.; Pandey, S.; Emura, T.; Hidaka, K.; Hossain, M. A.; Shrestha, P.; Sugiyama, H.; Endo, M.; Mao, H., Decreased water activity in nanoconfinement contributes to the folding of G-quadruplex and i-motif structures. Proc. Natl. Acad. Sci. U S A 2018, 115 (38), 95399544.

S5. Mills, J. B.; Vacano, E.; Hagerman, P. J., Flexibility of single-stranded DNA: use of gapped duplex helices to determine the persistence lengths of poly(dT) and poly(dA). J. Mol. Biol. 1999, 285, 245-257.

S6. Record, M. T. J.; Anderson, C. F.; Lohman, T. M., Thermodynamic analysis of ion effects on the binding and conformational equilibria of proteins and nucleic acids: the roles of ion association or release, screening, and ion effects on water activity. Quart. Rev. Biophys. 1978, 11, 103-178.

S7. Shlyakhtenko, L. S.; Potaman, V. N.; Sinden, R. R.; Lyubchenko, Y. L., Structure and dynamics of supercoil-stabilized DNA cruciforms. J Mol Biol 1998, 280, 61- 72.

S8. Jarzynski, C., Nonequilibrium Equality for Free Energy Differences. Phys. Rev. Lett. 1997, $78,2690-2693$.

S9. Zuker, M., Mfold web server for nucleic acid folding and hybridization prediction. Nucleic Acids Res. 2003, 31, 3406-3415. 
S10.Koirala, D.; Punnoose, J. A.; Shrestha, P.; Mao, H., Yoctoliter thermometry for singlemolecule investigations: A generic bead-on-a-tip temperature-control module. Angew. Chem. Int. Ed. Engl. 2014, 53 (13), 3470-3474.

S11.Doye, J. P. K.; Ouldridge, T. E.; Louis, A. A.; Romano, F.; Šulc, P.; Matek, C.; Snodin, B. E. K.; Rovigatti, L.; Schreck, J. S.; Harrison, R. M.; Smith, W. P. J., Coarse-graining DNA for simulations of DNA nanotechnology. Physical Chemistry Chemical Physics 2013, 15 (47), 20395-20414.

S12.Miyoshi, D.; Karimata, H.; Sugimoto, N., Hydration Regulates Thermodynamics of GQuadruplex Formation under Molecular Crowding Conditions. J. Am. Chem. Soc. 2006, 128 (24), 7957-7963. 